\title{
Jim Zhang: environmental health is a rewarding career to pursue!
}

Submitted Oct 26, 2018. Accepted for publication Oct 29, 2018.

doi: $10.21037 /$ jtd.2018.10.109

View this article at: http://dx.doi.org/10.21037/jtd.2018.10.109

\section{Editor's note}

Air pollution has been recognized as a major cause of death and disease globally. It poses health effects that lay burden on hospital admissions, emergency visits and increase the risk of premature deaths. According to the statistics of World Health Organization (WHO), an estimated 4.2 million premature deaths globally are associated with air pollution, which accounts for deaths and disease from lung cancer, stroke, ischaemic heart disease, acute respiratory infection and chronic obstructive pulmonary disease (1). The situation is indeed alarming and has to be dealt with by our joint efforts and unceasing actions.

As the Professor of Global and Environmental Health at Nicholas School of the Environment and Duke Global Health Institute, Duke University, Prof. Jim Zhang has been researching into the field of environmental health for decades with focuses on the development of novel biomarkers of human exposure and health effects, assessment of health and climate co-benefits of air pollution interventions, and examination of biological mechanisms by which environmental exposures exert adverse health effects. Fournal of Thoracic Disease (FTD) is honoured to have invited Prof. Zhang this time for the following interview in which he will share with us some of his research findings, insights, thoughts as well as some interesting stories behind the scene.

\section{Expert introduction}

Junfeng (Jim) Zhang currently serves as the Professor of Global and Environmental Health at Nicholas School of the Environment and Duke Global Health Institute, Duke University, Durham, North Carolina (Figure 1). He is also the Director of Regional Ozone Sino-US Collaborative Research Center as well as a Research Professor at Duke Kunshan University, Kunshan, Jiangsu Province, China.

Prof. Zhang's research interests include developing novel biomarkers of human exposure and health effects, assessing health and climate co-benefits of air pollution interventions, and examining biological mechanisms by

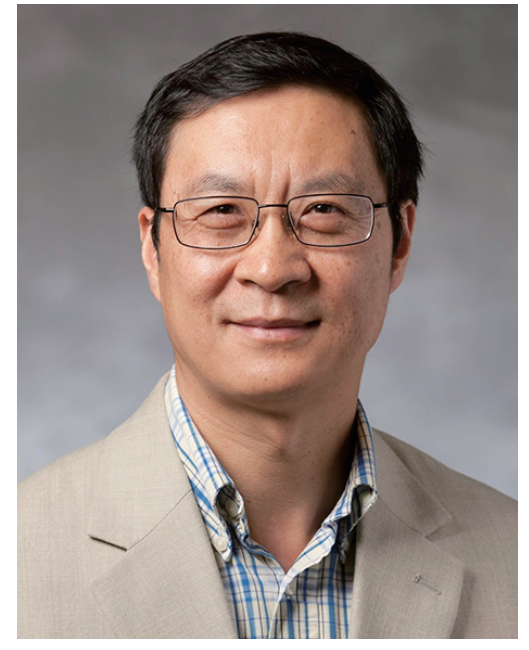

Figure 1 Prof. Jim Zhang.

which environmental exposures exert adverse health effects. He has led a number of international collaborations to study air pollution health effects and underlying pathophysiologic mechanisms. Recently he has been leading several studies to examine the efficacy of reducing indoor concentrations of PM2.5 via air filtration/purification in improving respiratory and cardiovascular health of adults and children.

Prof. Zhang has published nearly 200 peer-reviewed articles including those in top medical journals (the New England Fournal of Medicine, the Lancet, FAMA, 7AMA Internal Medicine, American fournal of Respirtaory and Critical Care Medicine) and those in top environmental science journals (Environmental Health Perspectives, Environmental Science and Technology). His $\mathrm{H}$ index is 58 . His work has been featured in major international media such as the Time, the New York Times, BBC, ABC, CBS, Yahoo News, etc. His early work on characterizing sources of non-methane greenhouse gases made him one of the officially recognized contributor to the 2007 Nobel Peace Prize awarded to IPCC. He is the 2012 recipient of the Jerome Wesolowski Award, the highest award of the International Society of Exposure Science. In 2013, he was elected as Fellow of American Association of Advancement in Science (AAAS). 


\section{Interview}

FTD: Having been examining air pollution health effects and underlying pathophysiologic mechanisms, what do you think are the critical issues facing the field?

Prof. Zhang: There are two major critical issues that need to be addressed. The first is what are the policy relevant studies and the second is what are the clinical/ prevention relevant studies. These issues are drastically different for the US and other more developed countries and for China and other developing countries. In the US, when recent and emerging studies show adverse health outcomes associated with air pollution exposure at levels below the current United States Environmental Protection Agency (EPA) standards, it is challenging to ascertain that the association is causal, due to the "background noise" at low concentrations. In China, when primary pollutants such as primary PM10 and PM2.5 and $\mathrm{SO}_{2}$ are declining, secondary pollutants such as ozone and organic aerosols are increasing or becoming more important. It is imperative but also challenging to understand what constituents of the air pollution mixture are most responsible for adverse health effects. (Understanding this can help set up most cost-effective control policies.) The second issue is about what advice can be given to the public to reduce the impact of air pollution exposure. In China, when outdoor air pollution levels are high, people are advised to stay indoors. This advice is only useful if effective indoor air purification device is used, including the addition of high-efficiency particulate air (HEPA) filtration in building's ventilation system or the use of portable air purifiers. In order for air purification devices to work effectively, the building must be kept air tight with windows and doors closed. In contrast, in the US, indoor air quality is often worse than outdoor air quality. Keeping building more air tight would worsen indoor air quality and hence to increase people's exposure.

\section{FTD: In a recent study, your team examined the relationship between free and total malondialdebyde (MDA), a well-established marker of oxidative stress, across various types of human biospecimens. Why is it important to study these MDAs?}

Prof. Zhang: Oxidative stress is involved in many diseases and in natural aging. MDA is a biomarker of oxidative stress, resulting from lipids peroxidation and can be readily measured in various biological specimens such as blood, urine, lung fluid, and exhaled breath condensate. It is relatively inexpensive to measure MDA. However, MDA can bound to many biomolecules such as proteins. Hence, MDA is present in free (unconjugated) and conjugated forms in biospecimens. Either form of MDA has been used in previous studies; but it is unknown which form is a better biomarker.

\section{FTD: What were the methodologies and major findings} of this study? What insights does this study bring to guide future studies?

Prof. Zhang: In my team's study, we examined the relationship between free and total (free plus conjugated) in urine, exhaled breath condensate, and serum, respectively. We found that in exhaled breath condensate, free MDA is the dominant form, which can be used to measure oxidative stress in the lung. In contrast, conjugated MDA is the dominant form in the serum, whereas free and conjugated MDA are moderately correlated in the urine. Our findings can help future studies to select the proper form of MDA.

FTD: The spectrum of your research is broad (e.g., cardiovascular disease, infectious diseases, children, and pregnant women, etc.). Which area/group of people do you think environmental exposures exert most adverse bealth impacts on?

Prof. Zhang: It is generally true that age and pre-existing disease are important susceptibility factors for air pollution health effects. Children, older people, and people with cardio-respiratory disease would be more vulnerable. However, studies also found adverse cardio-respiratory effects of air pollution in younger and healthy adults while not in older or those with chronic diseases. The explanation is that disease conditions or the medication use may have overwhelmed the effects of air pollution in those thoughtto-be more susceptible individuals.

FTD: We realize you are currently working on the project "Potential Pathophysiologic Mechanisms Linking Air Pollution Exposure in Pregnant Women to Reduced Birth Weight" funded by National Institute of Environmental Health Sciences (NIEHS). Would you introduce us to this project?

Prof. Zhang: Globally, outdoor air pollution has been deemed a significant health risk contributing annually to 3.2 million premature deaths worldwide and 76 million 
years of healthy life lost. These estimates are based on cardiorespiratory effects, although there is increasing evidence that air pollution affects other health outcomes including reproductive outcomes such as preterm birth and birth weight. My research team found that when ambient concentrations of PM2.5 and co-pollutants decreased by 13-60\% during the 2008 Beijing Olympics, babies born to pregnant women living in Beijing whose 8th month of pregnancy was during this short 47-day period of lower pollution levels, were $23 \mathrm{~g}$ (95\% CI: 5-40 g) larger than babies born to pregnant women with their 8 th month of pregnancy during the same calendar dates in 2007 or 2009 (when air pollution levels were higher). Such findings point to the importance of studying biological mechanisms by which air pollution may lead to reduced birth weight in order to provide data on which to help judge if a causal relationship between air pollution and birth weight exists. My team hypothesized that air pollution exposure leads to maternal inflammation, placental inflammation, altered placental development and transport capabilities, and fetal inflammation, resulting in reduced nutrient delivery to the fetus. Imprinted genes that play a substantial role in placental function may also be impacted, resulting in impaired regulation of nutrient exchange. These all may adversely impact fetal growth and thus reduced birth weight. Our multi-disciplinary team, covering expertise in obstetrics/gynecology, neonatology, epidemiology, epigenetics, exposure science, and biostatistics, is conducting this National Institutes of Health (NIH)funded study to test the hypotheses in a cohort of $>600$ pregnant Beijing women by measuring biomarkers reflecting mechanistic pathways pertinent to fetal growth in maternal blood and urine, cord blood, and placental tissue. We also measure internal doses of air pollution exposure and estimate external exposures to air pollution during pregnancy. Currently we are in the 2 nd year of this five-year study and have recruited some 60 women into the study. My role is one of the two multiple principal investigators.

\section{FTD: What are the most challenging aspects of research? Can you share with us a memorable case of yours?}

Prof. Zhang: The most challenging aspects of research have been funding constraints in the United States. The funding environment in the US has been very challenging in the last 10 years with NIH funding rate for investigator-initiated proposals (R01) lower than $10 \%$. The tough competition made those high-risk high-gain studies very difficult to be funded. The most memorable things out of my research have always been the fun times that I have had with my research team (collaborators and students). Especially by collaborating with clinicians on many of my projects, I have enjoyed learning clinical knowledges, given that I am not an MD but interested in investigating the impact of air pollution exposure on clinically relevant outcomes.

\section{FTD: Out of the variety of medical disciplines, why are you particularly interested in the study of environmental bealth?}

Prof. Zhang: My undergraduate training is chemistry and I also obtained a master's degree in atmospheric chemistry before pursuing a $\mathrm{PhD}$ degree in environmental health. I found it very interesting and rewarding to connect physical sciences and biological sciences in addressing health problems related to environmental factors. As a society, we have focused too much on treating diseases but too little on preventing diseases. The goal of environmental health is to improve public health. Making everyone heather is a rewarding career to pursue.

\section{FTD: As a world-renowned expert, what would be your tips to young researchers who would like to develop their expertise/career in your field?}

Prof. Zhang: Environmental health is an applied field of science. My advice would be to have an open mind and to find new knowledge that can be used to minimize health risks. Along my career path, multi-disciplinary collaborations have benefited me enormously. At the early stage of my career, I have learned from senior colleagues not only on how to do science but also how to work with others and to effectively manage projects. My wonderful mentors have been true role models for me to follow their successful steps. I would urge young researchers to find role models and follow their advice and wisdom.

\section{Acknowledgements}

We would like to express our sincerest gratitude to Prof. Jim Zhang for sharing his insights and opinions with us.

\section{Footnote}

Conflicts of Interest: The author has no conflicts of interest to declare. 


\section{References}

1. World Health Organization. Ambient air pollution:

Health impacts. Available online: https://www.who.int/

Cite this article as: Li B. Jim Zhang: environmental health is a rewarding career to pursue! J Thorac Dis 2018;10(12):E839E842. doi: 10.21037/jtd.2018.10.109 airpollution/ambient/health-impacts/en/

(Science Editor: Brad Li, JTD, jtd@amepc.org) 